treated, it seems possible that AMIC is under-recognized and may run undiagnosed as an ill-defined heart failure syndrome. Specific cardiac biomarkers may identify patients at risk. We sought to investigate the prevalence and associated factors for such biomarkers in SLE.

Methods One hundred sixty-eight consecutive patients (153 females) attending the a large lupus clinic, without past history of cardiac disease (heart failure, coronary artery disease, valvulopathy etc.) and/or pulmonary hypertension, were enrolled. None had chest pain or electrocardiographic (ECG) abnormalities suggestive of acute coronary syndrome. Highsensitivity cardiac troponin I (cTnI) and B-natriuretic peptide (BNP) were measured simultaneously in serum and plasma samples, respectively. Patients were categorized according to normal or abnormal BNP and/or cTnI. For the assessment of the impact of AM duration on abnormal cardiac biomarkers, patients were divided in two groups according to the median duration of use, which was calculated at 5.6 years in the current cohort. Statistical analysis was performed with SAS 9.0 software; $\mathrm{p}<0.05$ was considered significant.

Results Sixteen patients (9.5\%) had elevated BNP and/or cTnI. Compared to subjects with normal biomarkers, they were older, had longer disease and AM use duration and more frequently persistent creatine phosphokinase (CPK) elevation (table 1). Multivariable regression analysis showed prolonged $\mathrm{AM}$ treatment (>5.6 years) and persistent CPK elevation to be important predictors for elevated cardiac biomarkers $[\mathrm{HR}=5.43,95 \% \mathrm{CI}=1.14$ to $25.9, \mathrm{p}=0.034$ and $\mathrm{HR}=4.62$, $95 \% \mathrm{CI}=1.22$ to $17.51, \mathrm{p}=0.024$, respectively]. Two patients were diagnosed with AMIC on endomyocardial biopsy; both had CPK and BNP/cTnI elevation.

Conclusions Approximately 9\% of SLE patients had elevated myocardial biomarkers, in the absence of prior cardiac disease or pulmonary arterial hypertension. Prolonged AM therapy and persistent CPK elevation conferred an increased risk for abnormal BNP and cTnI, which might predict AMIC.

\section{CS-15 ENDOTHELIAL PROGENITOR CELLS AS A PROMISING BIOMARKER OF SYSTEMIC LUPUS ERYTHEMATOSUS ACTIVITY}

\begin{abstract}
1,2Gonzalo Silveira*, ${ }^{2,3}$ Sabrina Ranero, ${ }^{1}$ Adriana Carlomagno, ${ }^{4}$ Martín Rebella,

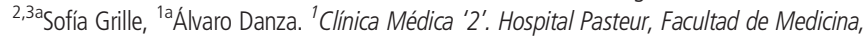
Universidad de la República, Montevideo, Uruguay; 'Departamento Básico de Medicina, Hospital de Clínicas, Facultad de Medicina, Universidad de la República, Montevideo, Uruguay; ${ }^{3}$ Cátedra de Hematología. Hospital de Clínicas, Facultad de Medicina, Universidad de la República, Montevideo, Uruguay; ${ }^{4}$ Clínica Médica 'C'. Hospital de Clínicas, Facultad de Medicina, Universidad de la República, Montevideo, Uruguay; ${ }^{a}$ Both authors contributed equally
\end{abstract}

\subsection{6/lupus-2018-Ism.50}

Background Endothelial progenitor cells (EPC) and circulating endothelial cells (CEC) are cell populations mobilized from the bone marrow in response to endothelial damage. Its relationship with vascular risk damage and subclinical vascular damage in systemic lupus erythematosus (SLE) is being actively studied, but their role as an activity biomarker was not evaluated.
Aim to study EPC and CEC levels in SLE patients and compare them to healthy controls, as well as study their relationship with clinical and analytical markers of disease activity.

Methods A prospective, analytical, case-control study was conducted; in a center for systemic autoimmune diseases in Montevideo, Uruguay. EPCs and CEC levels were quantified by multiparametric flow cytometry. EPCs were defined as $\mathrm{CD} 45^{\text {low } /}, \mathrm{CD}_{3} 4^{+}, \mathrm{CD}_{133^{+}}, \mathrm{CD} 31^{+}, \mathrm{CD} 146^{+}, \mathrm{CD}^{-}$and $\mathrm{CEC}$ as $\mathrm{CD} 45^{\text {low/-, }} \mathrm{CD} 34^{+}, \mathrm{CD} 133^{-}, \mathrm{CD} 31^{+}, \mathrm{CD} 146^{+}, \mathrm{CD} 3^{-}$.

Disease activity was assessed using 'Systemic Lupus Erythematosus Disease Activity Index' (SLEDAI) and 'Physician's Global Assessment' (PGA), erythrocyte sedimentation rate (ESR), C reactive protein (CRP), complement (C3 and C4), double-stranded DNA (dsDNA) antibody levels and proteinuria.

Results 28 controls and 22 SLE patients age and sex matched were enrolled. Patients median ages was $29.5 \pm 11.5$ years and controls were $36 \pm 11.25$ years, $(p=0.28)$. Sex ratio (female/ male) was $22 / 2$ and $24 / 4$ in patients and controls $(p=0.68)$.

SLE patients showed significantly lower levels of EPC (median $0.2202 \pm 0.4377 \mathrm{cells} / \mu \mathrm{l}$ vs $1.051 \pm 1.3849 \mathrm{cells} / \mu \mathrm{l})$, $\mathrm{p}<0.0001$, and lower levels of CEC (median 0.2321 \pm 0.49677 cells $/ \mu \mathrm{l}$ vs $0.7043 \pm 0.5706$ cells $/ \mu \mathrm{l}), \mathrm{p}=0.0004$ than controls.

EPC levels correlated with proteinuria/creatininuria ratio $(\mathrm{r}=0.5386, \mathrm{p}=0.0097)$ and with dsDNA level $(\mathrm{r}=0.5118$, $\mathrm{p}=0.0211)$ in SLE patients.

EPC level was higher in patients with significant proteinuria (median $0.58710 \pm 1.5086$ cells $/ \mu$ vs $0.11502 \pm 0.42442$ cells $/ \mu$ 1), $\mathrm{p}=0.0474$ (figure 1); and in patients with increased dsDNA level (median $0.5871 \pm 1.2562$ cells/ $\mu$ vs $0.1150 \pm 0.3504$ cells/ $\mu \mathrm{l}), \mathrm{p}=0.0249$.

No correlation was found with the levels of C3, C4, SLEDAI, as well as prednisone dose at the time of enumeration.

Conclusions EPS and CEC levels correlate with disease activity in SLE, particularly with renal involvement. Currently, we are enrolling more patients in order to confirm these results, but we postulate that, in the future, EPC may be used as a biomarker of disease activity.

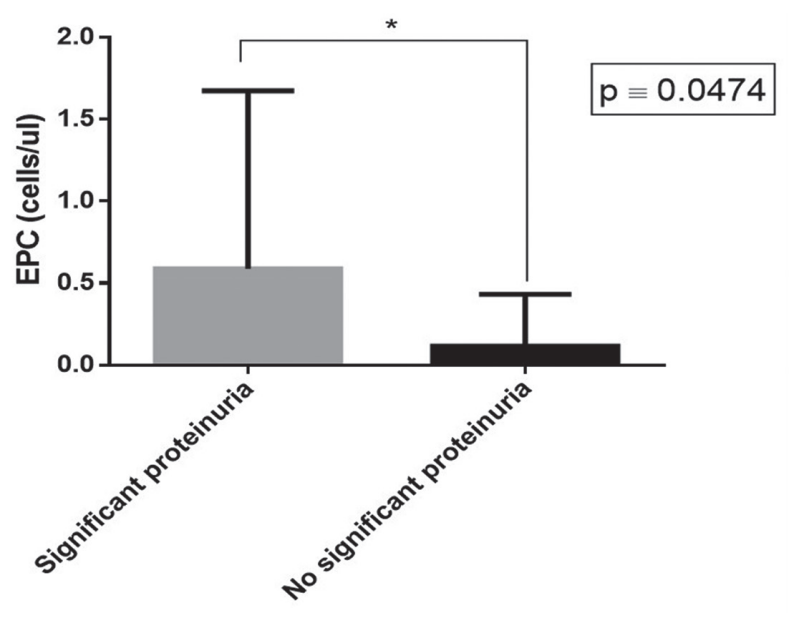

Abstract CS-15 Figure 1 EPC and significant proteinuria 